\title{
THE WRIST
}

D W Hodgkinson, N Kurdy, D A Nicholson, P A Driscoll

Most injuries to the wrist result from a fall on an outstretched hand
The wrist is composed of eight bones arranged in a proximal row (scaphoid, lunate, triquetrum, and pisiform) and distal row (trapezium, trapezoid, capitate, and hamate). Each row functions as a separate unit. Three articulations make up the carpus (figs 1 and 2).
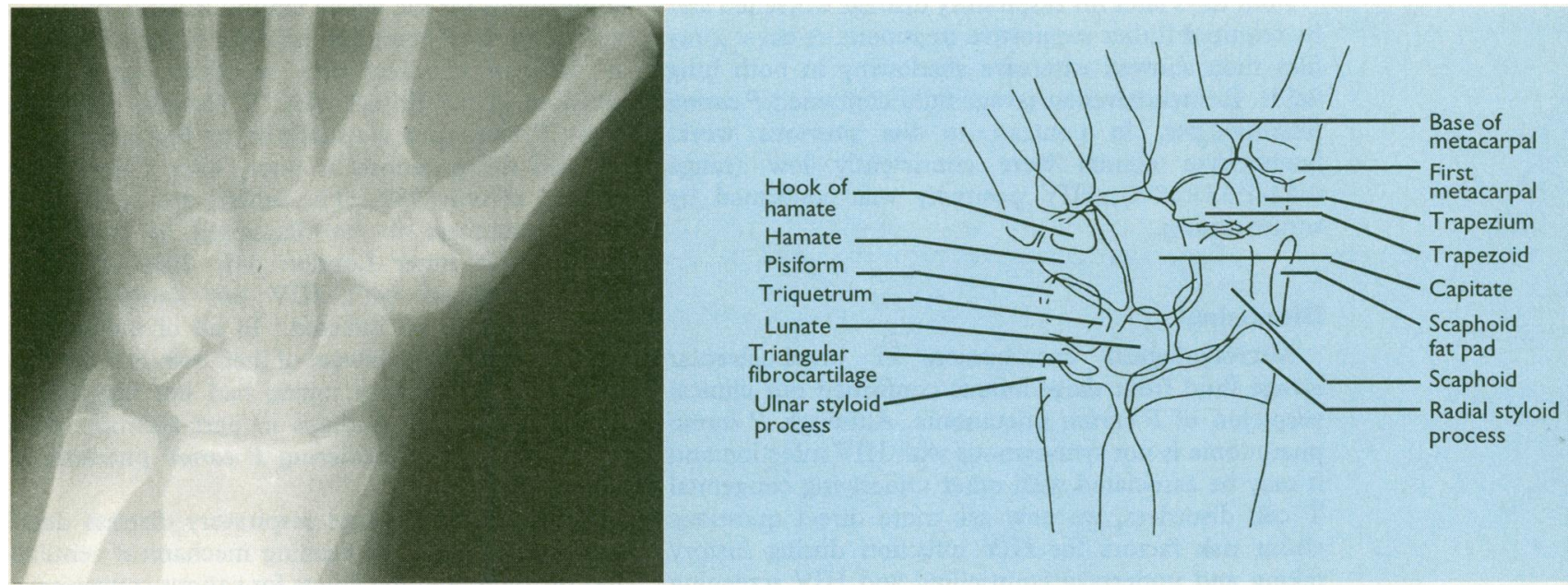

FIG 1-Normal posteroanterior radiograph of the wrist with line drawing showing anatomy.
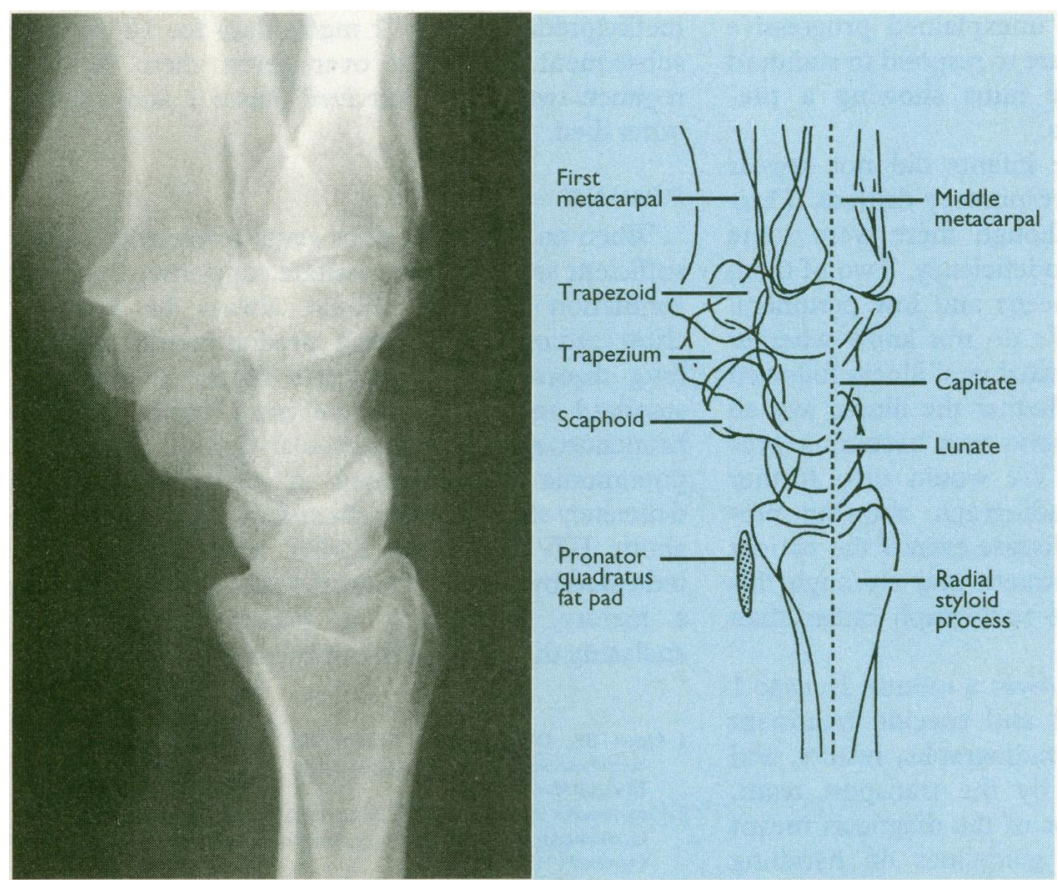

FIG 2-Normal lateral radiograph of the wrist with line drawing showing anatomy.
Radiocarpal joint - The distal radius articulates with the bones of the proximal carpal row (except the pisiform and triquetrum). This joint complex is supported by strong radiocarpal and intercarpal ligaments.

Carpocarpal joints-The proximal carpal row articulates with the distal row. These articulations are also supported by strong intercarpal ligaments that can be damaged in isolation after wrist sprains or in combination with scaphoid fractures and carpal dislocations.

Carpometacarpal joints-The distal carpal row articulates with the proximal end of the metacarpal bones. The finger carpometacarpal joints allow little movement compared with that of the thumb.

The carpal bones are bound to each other by short intercarpal ligaments, such as the scapholunate and the triquetrolunate. The articulation of the distal radioulnar joint including the triangular fibrocartilage allows pronation and supination at the wrist joint. 


\section{Development}

Primary ossification centres of the carpus begin to appear at 3 months of age, starting with the capitate and hamate. By the age of 5-6 years all the carpal bones have visible ossification centres. The last bones to ossify are the scaphoid and trapezoid.

\section{Mechanisms of common injury}

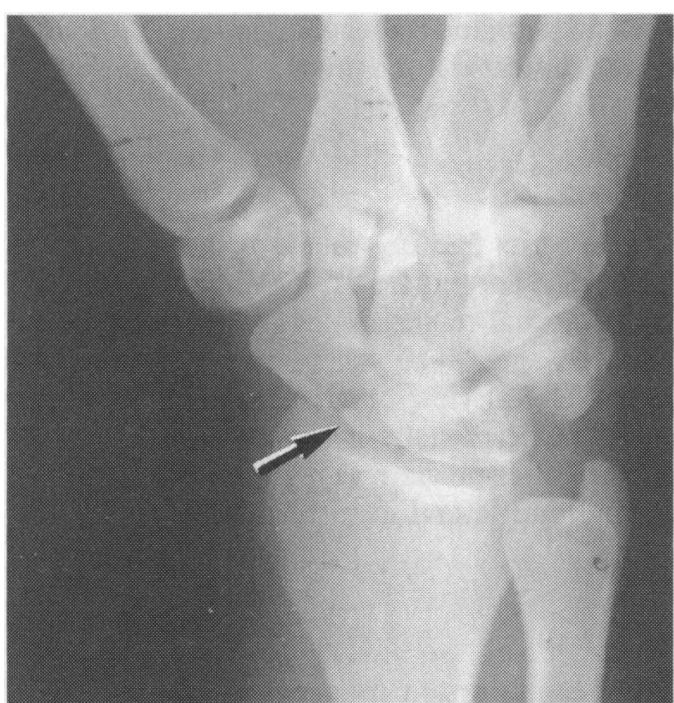

FIG 3-Oblique view of wrist showing a fracture through the waist of the scaphoid (arrow).

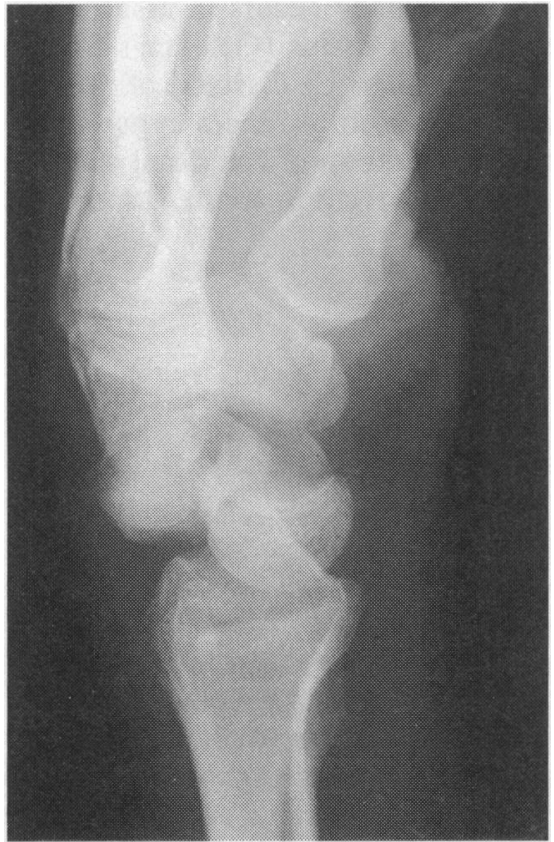

FIG 5-Lateral view of the wrist showing a perilunate dislocation.

Most injuries to the wrist are caused by a fall on the outstretched hand. This results in dorsiflexion of the hand and ulnar deviation of the hand together with supination of the carpus against a pronated forearm. The resultant force is focused across the waist of the scaphoid and the carpocarpal joint. The injury depends on the age of the patient, the severity and type of force, and the point of impact.

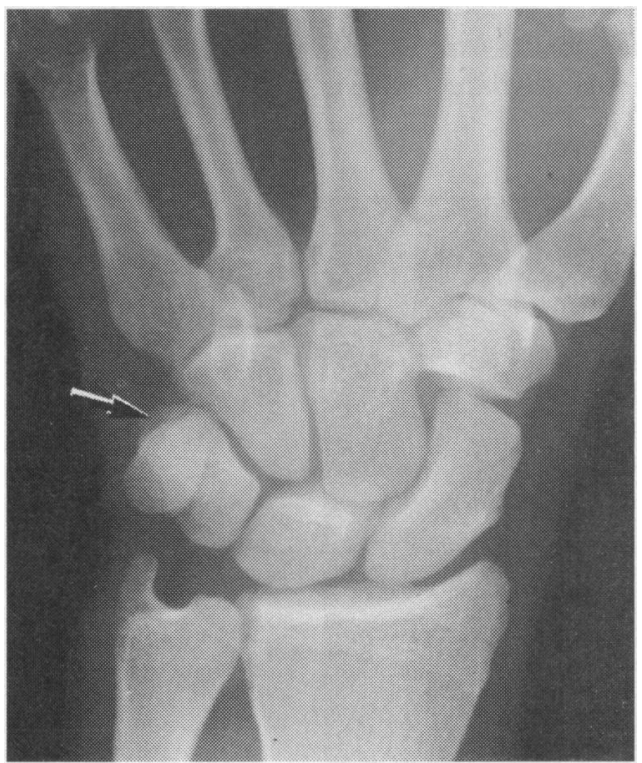
view showing a fracture of the triquetrum (arrow).

\section{Carpal fractures}

Wrist fractures usually occur in people aged $15-40$ years. The scaphoid is the most commonly fractured carpal bone (fig 3 ). Good quality scaphoid views will allow most scaphoid fractures to be identified from the initial films. If clinical suspicion remains, more specific imaging may be necessary. The triquetrum is the second commonest carpal bone to be fractured (fig 4).

\section{Carpal dislocations}

These are uncommon injuries but may be associated with a carpal fracture. The commonest carpal dislocations are the lunate, the lunate with a scaphoid fracture, and the perilunate (fig 5). The relationship of the lunate to the distal radius is best seen in the lateral view (fig 6).

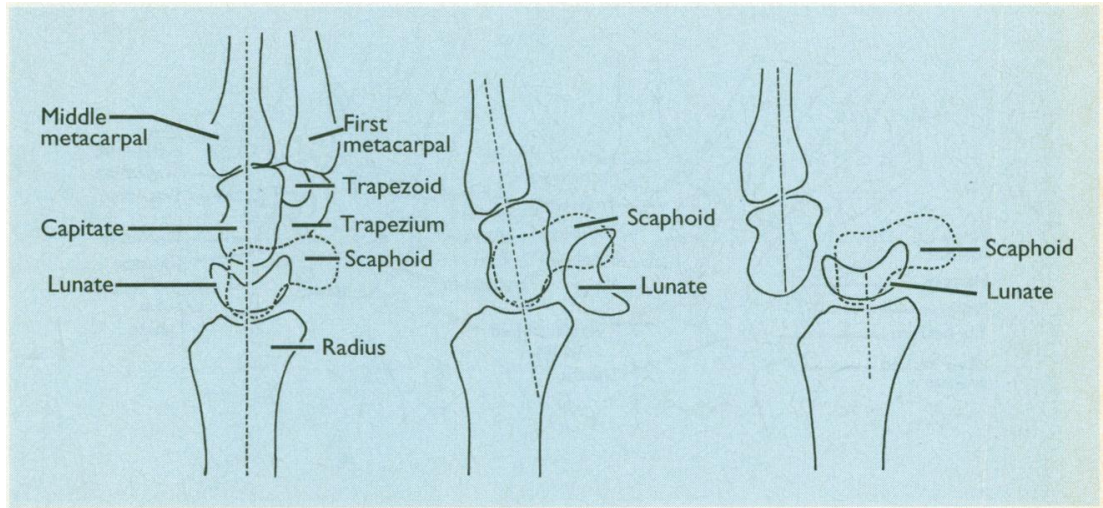

FIG G-Dislocations of the wrist. Left, normal lateral anatomy; middle, lunate dislocation; right, perilunate dislocation. 

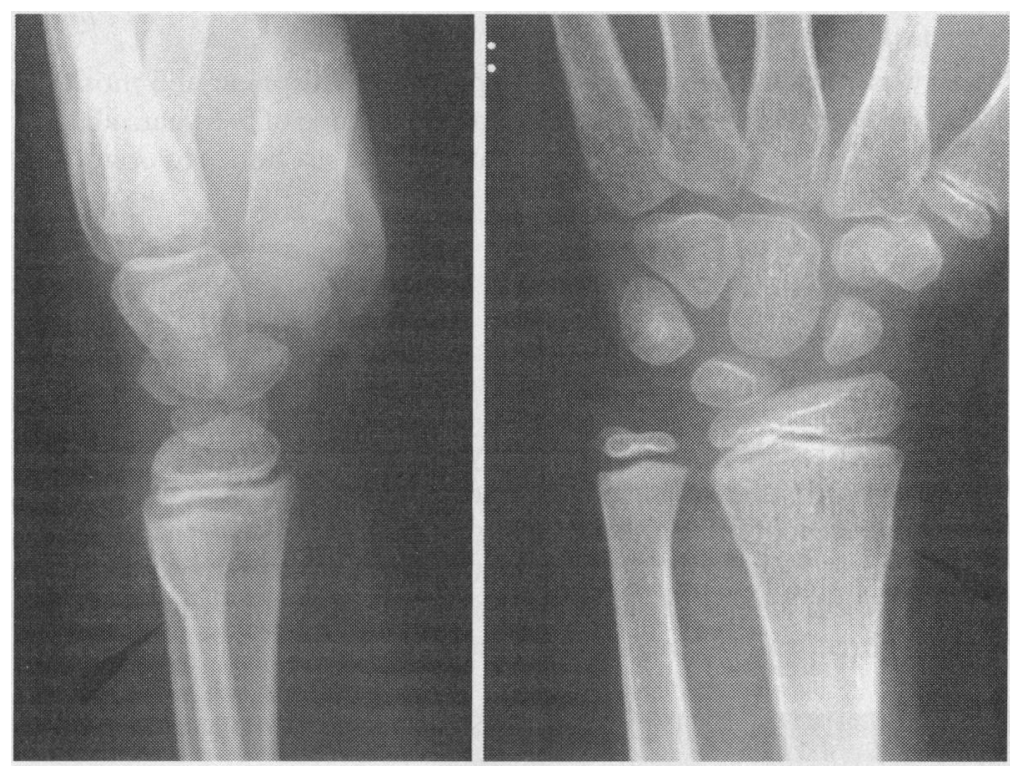

FIG 7-Lateral and posteroanterior of the wrist showing a torus fracture of the distal radial metaphysis (arrows).

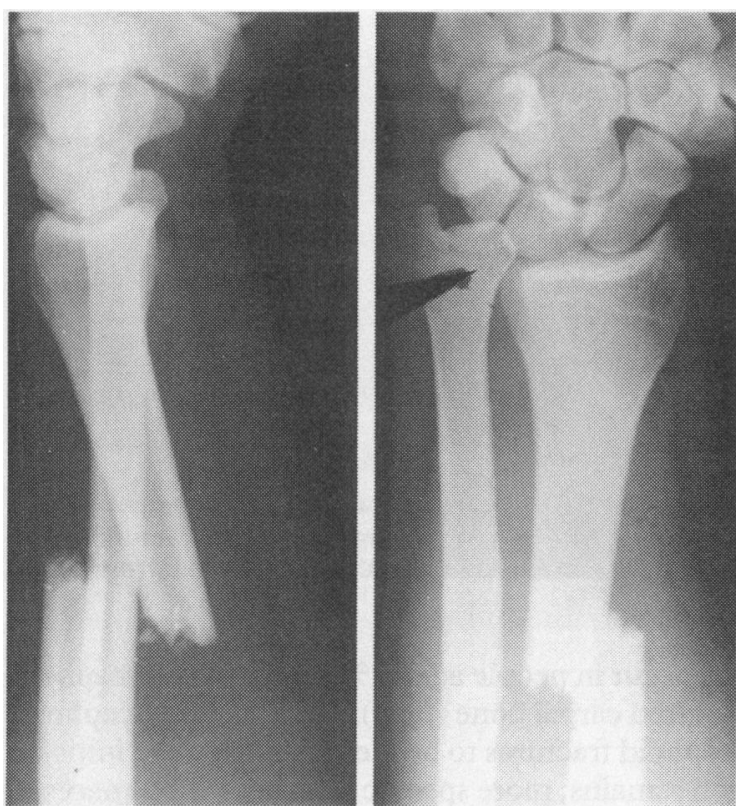

FIG 8-Lateral and posteroanterior wrist views showing Galeazzi's fracture dislocation. This is a fracture of the shaft of the radius with disruption of the distal radialulnar joint (arrow).

\section{Radiographic views}

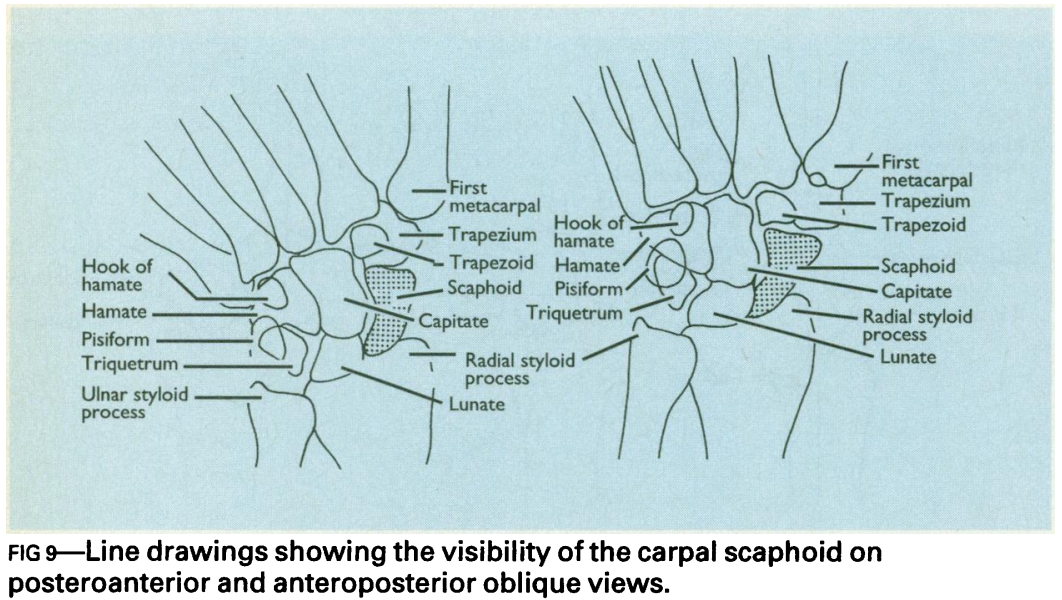

Fractures of the distal radius and ulna

Colles' fractures are common in middle aged and elderly women. Always look for other associated injuries such as concomitant scaphoid fractures. Epiphyseal and distal metaphyseal injuries are common in children. In most cases the fracture is evident. In children minimal greenstick fractures and epiphyseal injuries can be difficult to identify (look at the cortical margin, trabecular pattern, and soft tissue indicators of injury).

The torus fracture refers to the appearance of a buckled cortex (fig 7). This usually occurs $2-4$ $\mathrm{cm}$ proximal to the distal growth plate in children aged 4-10 years. It may be visible only in the lateral view on the dorsal surface of the cortex. Occasionally it can be seen in the posteroanterior view as a slight bump on the medial and lateral cortical margins.

Other important fractures in this region include Smith's fracture (which is opposite to a Colles' fracture and is differentiated from it in the lateral view) and Barton's fracture (a fracture-dislocation of the radiocarpal joint). An isolated ulnar styloid process fracture is rare. It usually occurs in association with the more common distal radial fractures. Isolated dislocation of the distal radioulnar joint is also uncommon but usually occurs in association with fracture of the radius (Galeazzi's fracture, fig 8). It is recognised by diastasis of the radioulnar joint, which produces apparent shortening or overriding of the distal radial articular surface in relation to the distal ulna.

Posteroanterior film-This includes the base of the metacarpals, the carpometacarpal joints, the carpocarpal joints, the radiocarpal joint, and the distal radius and ulna in profile.

The lateral view is essential for identifying dislocations of the carpus and common avulsion fractures of the dorsal surface of the triquetrum.

Posteroanterior oblique view-As well as the above projections the specific views for assessing the carpal scaphoid include the posteroanterior oblique and anteroposterior oblique, both taken with the wrist in ulnar deviation. These views maximise the visibility of the scaphoid bone and all four views are required for proper assessment (fig 9). 

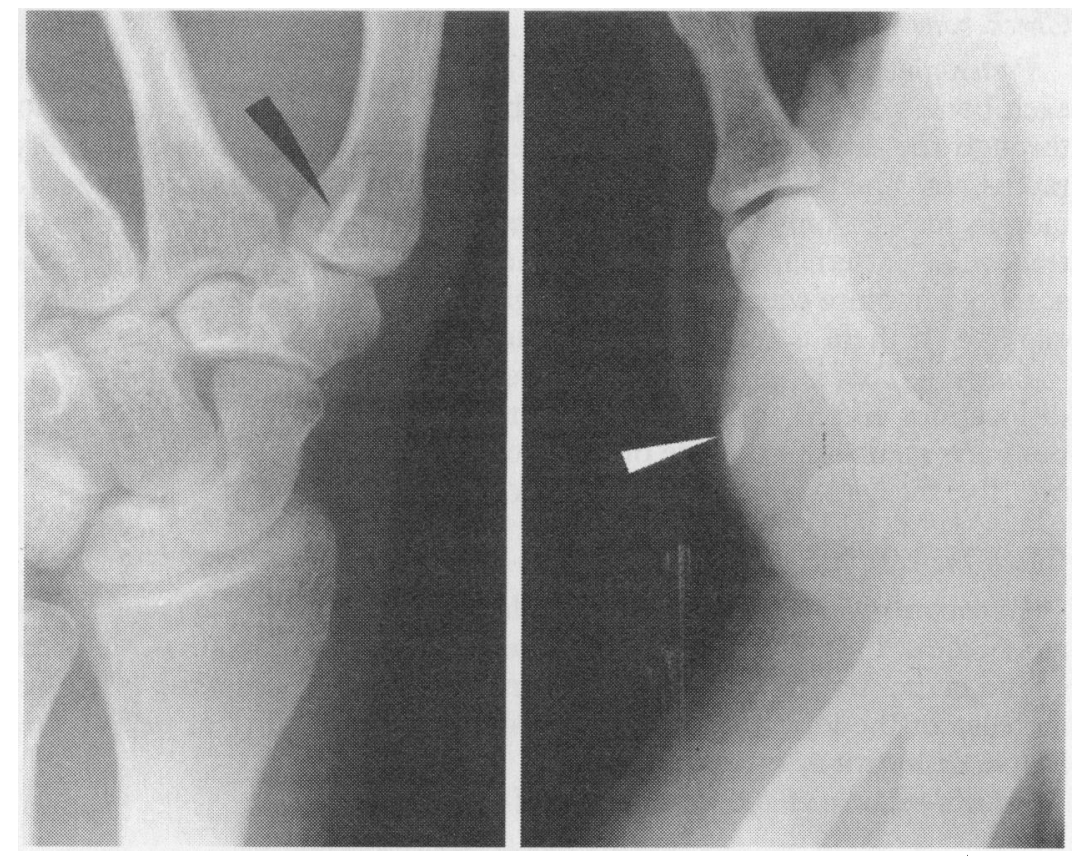

Tangential views may be used to detect foreign bodies (fig 10). More specific views of the wrist are available to suit particular problems but these would be requested only by a specialist.

FIG 10-Posteroanterior and tangential views showing glass (arrow).

\section{System of radiographic assessment}

\section{ABCs system of radiographic assessment}

Adequacy
Alignment
Bone
Cartilage and joints
Soft tissues

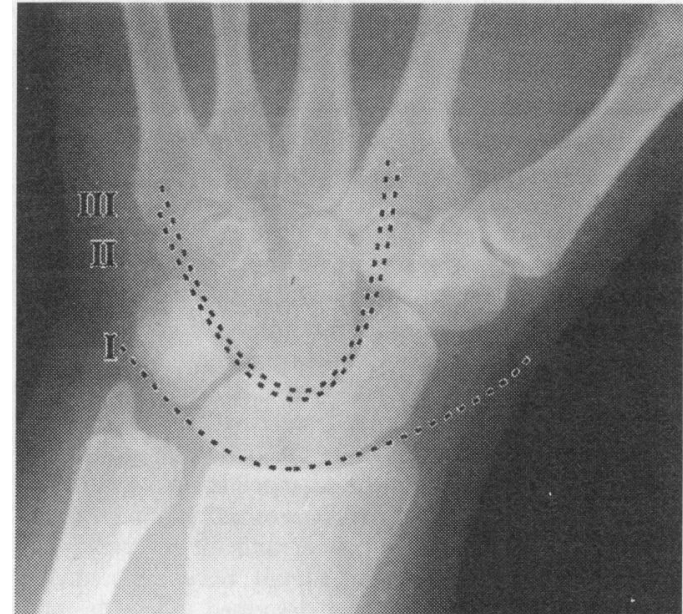

FIG 11-Three arcs joining the articular margins of the carpal bones

Tracing the outline of each bone can be difficult in the lateral view because many of them are superimposed

\section{Check the adequacy and quality of the radiograph}

Posteroanterior-Check for any rotation. There should be no overlap between the distal radius and ulnar bones. The trabeculae within the carpal bones should be visible and the bone margins should be clear and sharp. This is particularly important when assessing the carpal scaphoid, when subtle radiological signs may be obscured in poor quality films.

Lateral-Check for any rotation: the distal radius and ulna should be superimposed on each other, as should the bases of the metacarpal bones.

\section{Check alignment of bones}

Posteroanterior-The joint margins should be parallel and the width of the joints and distance between apposing carpal bones should be 1-2 mm. Excessive widening or narrowing indicates displacement of at least one of the adjacent carpal bones. In your mind draw three parallel arcs joining the articular margins of the carpal bones (fig 11). The first arc is formed by the proximal articular margins of the proximal carpal row (the scaphoid, lunate, and triquetrum), the second by connecting the distal articular margins of the proximal carpal row, and the third by joining the proximal articular margins of the capitate and hamate. Any disruption of these parallel lines indicates subluxation or dislocation of the carpal bone. The pisiform is normally superimposed on the triquetrum.

Lateral-There should be $10-15^{\circ}$ of volar angulation of the radiocarpal joint. The most important line to follow bisects the metacarpal base, the capitate, the lunate, and the distal radius (fig 6). The proximal pole of the capitate is inset within the lunate and the lunate in turn is inset within the radius. Look for evidence of carpal dislocation, which will disrupt this alignment. Also assess the alignment of the thumb metacarpal to the trapezium. 


\section{Scaphoid fat pad sign}

The scaphoid fat pad sign is positive if the fat is displaced laterally away from the concave surface of the scaphoid forming a linear or convex line
Swelling of the soft tissue may be the only radiological indication of an injury to the wrist

\section{Check bone margins and density}

Posteroanterior-Check the margins and internal trabecular pattern of each bone systematically. Start with the base of the metacarpals and work through the distal carpal row, proximal carpal row, and finally the distal radius and ulna. Look for evidence of disruption. In children the cortical margin may be only minimally disrupted, but disruption of the internal trabecular pattern may be more pronounced. When looking for a carpal scaphoid fracture check the bone margin of the articular cortex opposite the capitate. If there is no disruption, fracture is unlikely.

Lateral-This is the best view to detect carpal and metacarpal dislocations and fracture of the triquetrum and distal radius. Avulsion of a bone fragment from the site of insertion of the dorsal radiocarpal ligament into the triquetrum often occurs after flexion at the wrist.

\section{Check the cartilage and joints}

Posteroanterior-Check the distal radioulnar joint for evidence of diastasis as in Galeazzi's fracture. Then check the orientation and relationship of each of the carpal bones. Take particular note of the scapholunate joint. An increase in the joint space may indicate carpal instability or perilunate or lunate dislocation, or both.

Lateral-Look at the orientation of the lunate (fig 5).

\section{Check the soft tissues}

Posteroanterior-A bright light is needed to see soft tissue swelling. The scaphoid fat pad is a linear collection of fat located between the radial collateral ligament and the synovial tendon sheaths of the extensor pollicis brevis and the abductor pollicis longus. It is not seen consistently in children under 12 years old. A positive scaphoid fat pad sign is a useful indicator of injury on the radial side of the wrist. It should not be used alone to confirm the presence or absence of a scaphoid fracture. Check for foreign bodies. Tangential views may help visualisation by removing superimposed bone shadows (fig 10).

Lateral-An abnormal pronator quadratus shadow on the volar aspect of the wrist (anterior bowing $>1 \mathrm{~cm}$ from the volar cortical margin of the distal radius or obliterated) may be the only radiological sign of a distal forearm injury in children and adults (fig 2). This shadow is visible because of a thin layer of adipose tissue overlying the muscle belly.

D W Hodgkinson is senior registrar in emergency medicine, $\mathrm{N}$ Kurdy is tutor in orthopaedic surgery, D A Nicholson is consultant radiologist, and P A Driscoll is senior lecturer in emergency medicine, Hope Hospital, Salford.

The line drawings were prepared by Mary Harrison, medical illustrator.

The ABC of Emergency Radiology has been edited by David Nicholson and Peter Driscoll.
Meticulous clinical examination

Appropriate radiograph and systematic assessment

\section{ANY QUESTIONS}

When a woman who has suffered from von Recklinghausen's disease for over 20 years was prescribed ampicillin and flucloxacillin her skin swellings became smaller and softer. When the drug was stopped the lumps started to enlarge again. Is there any explanation for this?

Von Recklinghausen's disease is now usually referred to as neurofibromatosis type 1 . It is one of the commonest autosomal dominant genetic disorders, with an incidence at birth of about 1 in $3000 .^{1}$ The first signs of the disease develop in early childhood. These are multiple café au lait spots. The general population may have one or two of these spots, but people with neurofibromatosis type 1 have six or more. The spots may be associated with axillary freckling. As patients reach the teenage years they usually begin to develop small neurofibromas on the skin. These are soft, cutaneous swellings often with a purplish coloration. When they first develop they appear as slightly raised swellings, which are rarely painful, but as patients become older the neurofibromas become papillomatous. The number of neurofibromas is extremely variable, from just a few to many hundred.
They rarely cause symptoms apart from occasional itching. There are no known drugs which definitely influence the growth of neurofibromas in terms of number and size. The only thing that some female patients report is that during pregnancy the neurofibromas increase in size and sometimes in number. Some female patients report that they become less obvious once the baby has been born. Riccardi has reported that the mast cell inhibitor ketotifen may affect the growth of neurofibromas, ${ }^{2}$ although other workers are less convinced. It is difficult to know why ampicillin and flucloxacillin should have affected the patient's lesions in the manner described unless the skin over some of the lesions had actually become chronically infected and this is what resulted in the change in appearance. I have not heard other patients with neurofibromatosis type 1 report any changes in their neurofibromas while being treated with antibiotics.SUSAN M HUSON, consultant clinical geneticist, Oxford

1 Huson SM, Harper PS, Compston DAS. Von Recklinghausen's neurofibromatosis: a clinical and population study in south east Wales. Brain 1988;11:1355-81.

2 Riccardi VM. Neurofibromatosis. Phenotype, natural history and pathogenesis. 2nd ed. Baltimore: Johns Hopkins University Press, 1992:1-498. 